\title{
Comparison of evolutionary and swarm intelligence-based approaches in the improvement of peach fruit quality
}

\author{
A. Kadrani ${ }^{1}$, M.M. Ould-Sidi², B. Quilot-Turion ${ }^{3}$, M. Génard², F. Lescourret ${ }^{2}$ \\ ${ }^{1}$ INSEA, BP 6217 Rabat-Instituts, 10106 Rabat, Maroc \\ ${ }^{2}$ INRA, UR1115 Plantes et Systèmes de culture Horticoles, F-84914 Avignon, France \\ ${ }^{3}$ INRA, UR1052 Génétique et Amélioration des Fruits et Légumes, F-84143 Montfavet, \\ France
}

\begin{abstract}
We investigated two major families of algorithms for the multi-objective optimization: evolutionary and swarm intelligence-based optimization approaches. Non-dominated Sorting Genetic Algorithm II (NSGA-II) and multi-objective particle swarm optimization (MOPSO) algorithms are biology inspired and are population-based as use a set of solutions which evolve within the search space. These approaches employ different strategies and computational effort; therefore, a comparison of their performance is needed. This paper presents the application and performance comparison of NSGA-II and one variant of the MOPSO, namely MOPSO-CD which incorporates the crowding distance computation and the constraints handling, to design ideotypes for sustainable fruit production systems. The design of peach ideotypes that satisfy the requirement of high fruit quality and low sensitivity to brown rot in a given environment was formulated as a multi-objective problem, and both NSGA-II and MOPSO-CD are used to find the best combinations of genetic resources and cultural practices adapted to, and respectful of specific environments. Statistically significant performance measures are employed to compare the two algorithms.
\end{abstract}

Keywords: Multi-objective optimization, genetic algorithm, particle swarm optimization, decision-making.

\section{INTRODUCTION}

Modeling and simulation are increasingly used to tackle the complexity of agricultural production systems . Researchers have to propose innovative perspectives of evolution towards systems respectful of the environment and producing safe food while ensuring the economic viability of farms. They need to deal with strong genotype $\mathrm{x}$ environment $\mathrm{x}$ practices interactions to design best combinations of genetic resources and cultural practices adapted to specific agro-environmental conditions optimizing the trade-off between strongly antagonistic criteria. In this work, we deal with the case of brown rot which is one of the main diseases causing large economic losses for peach growers. The challenge is to build-up a tool to conceive innovative management strategies that optimize genotype $\mathrm{x}$ environment $\mathrm{x}$ practices interactions to limit fruit contamination by brown rot while keeping or improving fruit quality (sweetness and fruit fresh mass). Hence design of peach ideotypes for a given set of cultural practices and a specific environment is governed by a set of objective functions and constraints.

Several methods have been proposed to deal with multi-objective optimization problems. Two types of approaches can be distinguished: the aggregative approaches and the Pareto dominance based approaches. In this paper, we investigated two major families of the Pareto dominance algorithms: the Multi-Objective Evolutionary Algorithms (MOEAs) and the MultiObjective Particle Swarm Optimization (MOPSO). These two families are biology inspired and are population-based as use a set of solutions which evolve within the search space.

In recent decades, a wide range of MOEAs has been developed. One of the most cited MOEAs is the Non-dominated Sorting Genetic Algorithm II (NSGA-II). This success story of NSGA-II could be attributed, as announced by Deb, to its low computational requirements, its 
elitist approach, and its simple constraint-handling method. For these reasons, NSGA-II has become the reference for researchers in the field of MOEAs.

MOPSO are more recent algorithms which are based on biological metaphor. These algorithms are inspired and imitate the movement of birds, fishes, or others organisms in their research of food. Large number of MOPSO algorithms was proposed in the literature. The diversity maintenance mechanism of MOPSO while seeking global Pareto-optimal solutions is rather poor compared with other MOEAs such as NSGA II. This motivated researchers to introduce into MOPSO algorithms some procedures from the MOEAs such as elitism, diversity operators, mutation operators, constraint handling and crowding distance.

Raquel and Naval have proposed an algorithm that called MOPSO-CD which incorporates the crowding distance computation and the constraints handling of NSGA-II.

In this study, we choose to compare the performance of the NSGA-II with those of MOPSO$\mathrm{CD}$ in the case of the improvement of peach fruit quality. Both algorithms NSGA-II and MOPSO-CD were interfaced with the 'Virtual Fruit' process-based model which describes quality traits of peach fruit during the growth stage. The performance of each algorithm is analyzed qualitatively by comparing the non-dominated Pareto front and the performance measures.

The remainder of this paper is organized as follows. The modeling of the peach fruit quality with the problem formulation is discussed in Section 2. Section 3 presents a brief description of NSGA-II and MOPSO-CD. Section 4 provides the experimental results obtained by the two multi-objective algorithms and discusses their performance measures with a fuzzy logic based strategy for selection of a compromised solution.

\section{Case study description and mathematical formulation}

The brown rot is one of the main diseases causing large economic losses for peach growers. Today, no other alternative than chemical treatment is available so fungicide applications are generalized and occur till pre-harvest. Those practices are acceptable neither for environment nor for consumer health. We focus on the model-based design of sustainable peach ideotypes: combination of genetic material (genotypes) and cultural practices optimized for specific environment. We used the 'Virtual Fruit' a process-based model which simulates peach growth, to perform virtual experiments. In past work, six genetic parameters of the 'Virtual Fruit' were identified as the most important and genotype depending. These parameters are considered as the decision variables for our optimization problem and they are described as follows:

\begin{tabular}{|c|c|c|c|c|}
\hline $\begin{array}{l}\text { Variabl } \\
\mathrm{e}\end{array}$ & Unit & Definition & $\begin{array}{l}\text { Min } \\
\text { value }\end{array}$ & $\begin{array}{l}\text { Max } \\
\text { value }\end{array}$ \\
\hline & $\begin{array}{l}\text { dimensionles } \\
\mathrm{S}\end{array}$ & $\begin{array}{l}\text { Leaf structural mass/ leafy shoots structural } \\
\text { mass }\end{array}$ & 0.672 & 0.842 \\
\hline & degree day ${ }^{-1}$ & Initial relative fruit growth rate & 0.001 & 0.01 \\
\hline & dimensionles & $\begin{array}{l}\text { Proportion of carbon as sucrose in phloemic } \\
\text { sap }\end{array}$ & 0.23 & 0.54 \\
\hline & $\mathrm{g}$ & $\begin{array}{l}\text { Empirical coefficient relating stone dry mass } \\
\text { and total fruit dry mass }\end{array}$ & 6.1 & 7.46 \\
\hline & $\begin{array}{l}\text { dimensionles } \\
\text { s }\end{array}$ & $\begin{array}{l}\text { Parameter of the equation of the relative } \\
\text { expansion rate of the cuticle surface area }\end{array}$ & 1.134 & 1.386 \\
\hline & $\mathrm{gcm}^{-2} \mathrm{bar}^{-1} \mathrm{~h}^{-1}$ & Hydraulic conductance per unit of fruit surface & $\begin{array}{l}0.076 \\
9\end{array}$ & 0.667 \\
\hline
\end{tabular}

Table 1. Lower and upper bounds of the optimization variables 
The objective of our approach is to create virtual genotypes (combination of the six parameters) leading to fruits of good quality (large and sweet) with low skin density of cracks in order to limit sensitivity to brown rot. Therefore, three criteria simulated by the model were chosen: Fruit mass, fruit sweetness, and the density of cracks:

i. Fruit fresh mass remains the only criterion of payment for the peach producers. This criterion should be maximized with the range of the required mass in the market:

ii. Fruit taste represents an increasing economic challenge in order to answer a global social demand regarding fresh product quality. The fruit sweetness is then the second criterion to be maximized under reasonable values:

iii. Finally, we also considered the density of cracks of the fruit skin. This criterion may largely influence fruit sensitivity to brown rot which is one of the main polycyclic diseases in stone fruit and causes large economical losses. has to be minimized respecting some thresholds:

The bounds of variation of the three criteria were set to 50 (g) and 300 (g) for the fruit mass , $4 \%$ and $20 \%$ for sweetness and 0 and $20 \%$ for skin crack density . The normalization should be performed in order to take into account the constraints on criteria. Thus, the transformed objective functions will be defined in $[0,1]$ as follows:

where . The genotype design problem can be stated as follows:

where and are the bounds of the optimization variables (see Table 1).

\section{Multi-objective optimization approaches}

3.1. Multi-objective Evolutionary Algorithm: NSGA II

NSGA-II is one of the MOEAs, proposed by Deb et al. as a significant improvement to the original NSGA by using a more efficient ranking scheme and improved selection to capture the Pareto front. In NSGA-II, the selection process at various stages of the algorithm toward a uniformly spread-out Pareto optimal front is guided by assigning fitness to chromosomes based on domination and diversity. Domination is determined by ranking all chromosomes in the population, where chromosomes with higher rank are considered to have better fitness. Chromosomes with the same rank are compared based on their diversity which is defined based on a crowding measure for each chromosome. Chromosomes with larger values of crowding distance are preferred more to be selected for next generations. Interested readers may refer to Deb et al. for a detailed description of the algorithm. A brief step-by-step description of the NSGA-II pseudo-code is as follows:

1. Initialization: randomly initialize parameters of the
population of size $N$ ( $N$ individuals). This population is
called parent population and noted $P$.
2. Evaluation : calculate the values of criteria and
constraints for each individual


3. Classification: rank the population using non-domination relation (best individuals in the first front).

4. Preserving the diversity within the population: calculate the crowding distance. The crowding distance computes the relative closeness of a given solution to other solutions on the same front. The maximal the crowding distance the better the solution.

5. Child population: use genetic operators i.e. selection, crossover, mutation to create a child population from the parent population ( $N$ children). Child population is noted Q.

6. Evaluation of child population

7. Recombined population: combine the parent and child population and calculate the crowding distance. The recombined population noted $\mathrm{R}$ is of size $2 \mathrm{~N}$.

8. New parent population: select $\mathrm{N}$ best individuals based on the rank and the crowding distance. This population will be used as parent population $P$ in the next generation.

9. Go to the step 3 and repeat until stopping criterion is satisfied (number of generations in our case)

\subsection{Multi-objective Swarm Intelligence: MOPSO-CD}

MOPSO-CD is a variant of MOPSO that extends the PSO algorithm to handle MOPs by incorporating the mechanism of crowding distance computation into the algorithm of PSO specifically on global best selection and in the deletion method of an external archive of nondominated solutions. The diversity of non-dominated solutions in the external archive is maintained by using the mechanism of crowding distance together with a mutation operator which was proposed in MOPSO. Crowding distance computation, Global best selection and Mutation operation were incorporated to MOPSO-CD. The main algorithm consists of initialization of the population, evaluation and reiterating the search on swarm by combining PSO operators with Pareto-dominance criteria. The non-dominated solutions found are stored in an external repository (ERP), and are used to guide the search particles. Whenever this archive (external repository) is full, it will be truncated using crowding distance mechanism. At the end of the execution, all the particles stored in the external archive give us an approximation of the true Pareto-optimal front. The pseudo code of MOPSO-CD is given below. More details of this algorithm can be found in .

1. Initialize swarm and size of external archive

2. Evaluate objective functions

3. Store pbests

4. Store non-dominated particles in external archive Iter $=0$

5. While(Iter<IterMax); where IterMax is the maximum number of iterations

o Compute the crowding distances in external archive

o Select the global best guide (using crowding distance)

o Update velocity and positions of the particles

o If $($ Iter<(PMut $x$ IterMax)); where PMut is the probability of mutation

Perform mutation 


\author{
End If \\ o Evaluate objective functions \\ o Update external archive \\ o Update pebsts \\ o Iter $=$ Iter +1 \\ End While
}

6. Report results (external archive)

\title{
4. Results and discussion
}

The 'Virtual Fruit' process-based model is linked to multi-objective optimization approaches to design genotypes adapted to cultural practices. The model runs on daily basis and it is driven by its parameters, which are constant over time and independent of the environment. The simulations were performed from 87 days after bloom (DAB) to 150 DAB in the climate of Avignon in 2009, and the cultural scenarios chosen was the low crop (LC) load combined with well-irrigated (WI) regime. The low crop load contains 4 fruits per fruit-bearing stem and is defined in the initial state of the model.

The NSGA II and MOPSO-CD are applied to the multi-objective problem presented above to generate the best combinations of the six parameters of the 'Virtual Fruit' model that maximize fruit mass and sweetness and minimize skin crack density. The optimization is performed at fruit maturity.

The initial population chosen for the both algorithms is 400 and the number of the generations is set to 200. Each candidate solution consisting of six parameters of the 'Virtual Fruit' is represented by a particle in MOPSO-CD and a chromosome in NSGA-II. The population is initialized randomly using $\mathrm{C}$ function and set as the initial population for both algorithms. After several experiments with different simulation parameters of each algorithm, the parameters listed in Table 2 are found to provide the best performance.

\begin{tabular}{|l|l|}
\multicolumn{1}{|c|}{ MOPSO-CD } & \multicolumn{1}{c|}{ NSGA-II } \\
\hline \hline Cognitive and social acceleration=1 & $\begin{array}{l}\text { Crossover probability=0.9 } \\
\text { Distribution parameter (for } \\
\text { Inertia weight }=0.9\end{array}$ \\
crossover)=20 \\
Mutation probability $=0.1$ \\
Archive size $=400$ & $\begin{array}{c}\text { Distribution parameter (for } \\
\text { mutation) }=0.5\end{array}$ \\
\hline
\end{tabular}

Table 2. Parameters of NSGA-II and MOPSO-CD algorithms

The two optimal Pareto fronts obtained in one of the 24 experiments using MOPSO-CD and NSGA-II algorithms are shown in Fig.3 for qualitative comparison purpose. Both algorithms have generated large numbers of solutions and it can be seen that the Pareto optimal front is showing a nonlinear relationship between the three objectives. The solutions provided to the decision maker are reasonable in terms of the three fitness functions. To check the performance of the multi-objective algorithms, various performance measures for evaluating a set of non-dominated solutions have been proposed in the literature. For the purpose of this paper, we use two metrics, namely set coverage metric (SC) and spacing metric (SP) , to measure performance of NSGA-II and MOPSO-CD. The set coverage metric gives the relative convergence and domination of solutions between two non-dominated sets and. The metric calculates the proportion of solutions in , which are weakly dominated by solutions of. means that all solutions in are weakly dominated by, while represents the situation when none 
of the solutions in are weakly dominated by. It may be noted that is not necessarily equal to . If this means that the set has better solutions than the set.

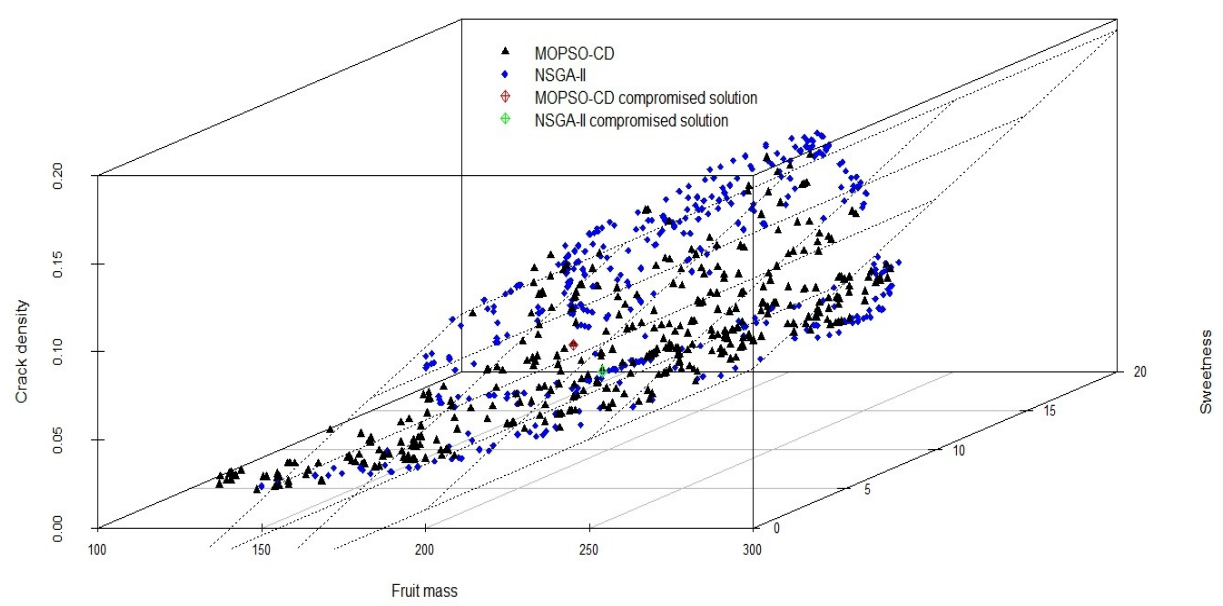

Figure 1. Optimal Pareto front obtained in one of 24 experiments using NSGA-II and MOPSO-CD.

The spacing metric was proposed as a way of measuring the homogeneity of the front description by computing the mean distance between each element of the Pareto estimation. This indicates how far the generated non-dominated solutions are closer and equidistantly spaced. A null value of SP means that the non-dominated solutions found are equidistantly spaced.

Table 3 shows the statistical summary of different performance metrics for both MOPSO-CD and NSGA-II algorithms based on 24 independent runs.

\section{Performance metrics}

Set coverage Spacing metric metric Statistic

\begin{tabular}{|l|l|l|r|l|}
\hline \hline Best & \multicolumn{1}{|l|}{0.700625} & 0.00354167 & 0.02107373 & 0.03020891 \\
Worst & 0.656875 & 0.000625 & 0.02692485 & 0.03020891 \\
Mean & $\mathbf{0 . 6 7 7 3}$ & 0.00175347 & $\mathbf{0 . 0 2 3 9}$ & 0.02974282 \\
Variance & $\mathbf{4 8 5 8}$ & & $\mathbf{7 1 2 4}$ & \\
SD & 0.0001769 & $1.0768 \mathrm{E}-06$ & $2.39718 \mathrm{E}-06$ & $3.67896 \mathrm{E}-06$ \\
& & & & \\
& & & & \\
\end{tabular}

Table 3. Summary statistics of performance metrics for the results of 24 runs. Bold numbers indicate the best performing algorithm.

This table indicates that NSGA-II provides the best results with respect to the set coverage metric, since the average value of is higher than the average value of (here is NSGA-II and is MOPSO-CD). Thus, in this case, NSGA-II is performing better than MOPSO-CD. NSGA- 
II also provides the best results of the SP metric in comparison with the MOPSO-CD. It can be observed that the mean value of the spacing metric for NSGA-II is lower than for MOPSO-CD. This indicates that the distribution of Pareto optimal solutions is closer equidistantly distributed in NSGA-II than in MOPSO-CD. Thus NSGA-II results in better performance.

Upon having the Pareto optimal set of non-dominated solutions, it is preferred to get the best compromise solution for implementation. There are many ways to select the compromise solution. In this study for final decision-making a fuzzy set-based approach is adopted. The fuzziness is defined by membership functions which represent the degree of fuzziness in some fuzzy set using values in . means that the membership is entirely not in the set, means completely in the set, and a number in between and means partially in the set. Membership value for th objective of a solution in the final Pareto front is represented by a membership function defined by

suggests how far the non-dominated solution is able to satisfy the objective. The accomplishment of each non-dominated solution with respect to all the non-dominated solutions can be obtained as follows:

The solution with maximum of value of is the compromised solution that can be chosen as the best solution or the one having the highest cardinal priority ranking.

Using the fuzzy set-based approach, the best compromised solution is found at points and on the Pareto fronts respectively generated by NSGA-II and MOPSO-CD (see Figure 1). Figure 2 shows the three fruit traits corresponding to the compromised solutions obtained using NSGA-II and MOPSO-CD.
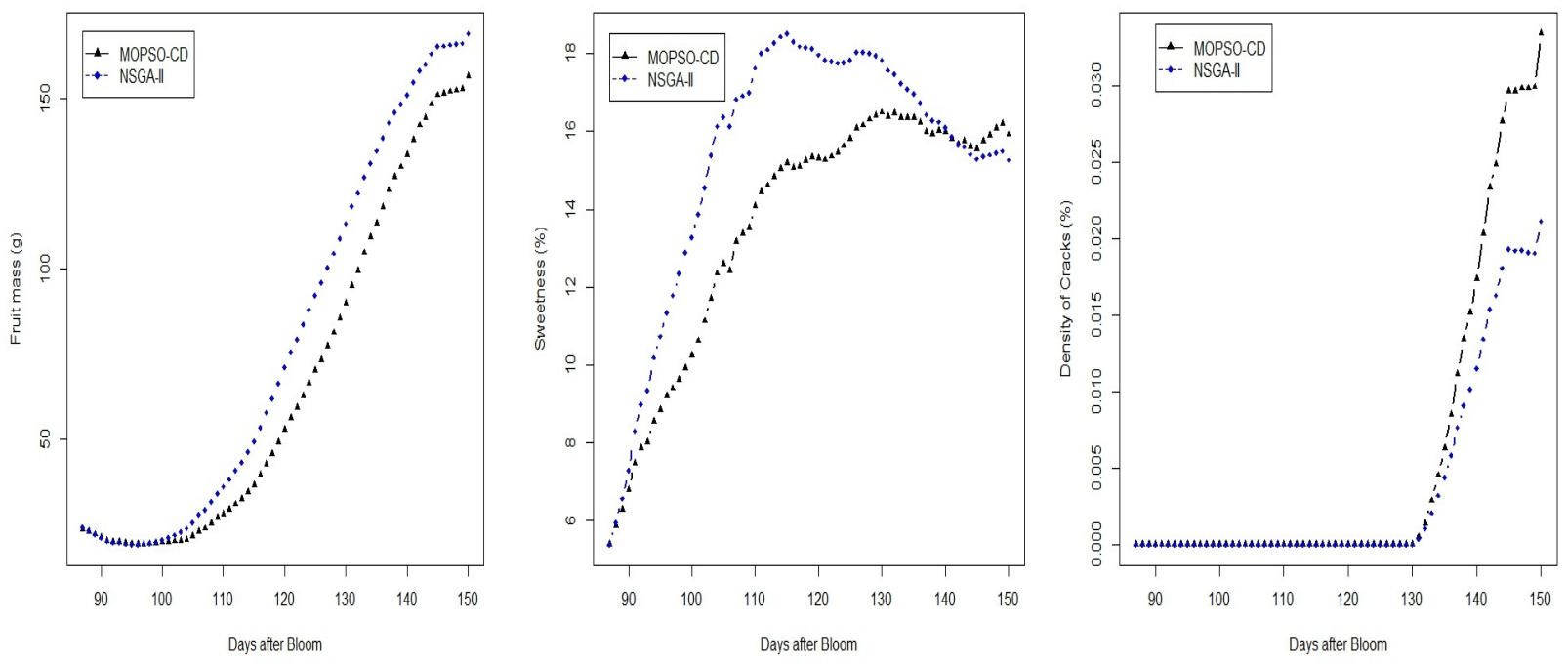

Figure 2. Simulation of the three fruit traits with the compromised solutions obtained using NSGA-II and MOPSO-CD algorithms.

\section{Conclusion}

In this paper, a multi-objective evolutionary algorithm (NSGA-II) and a multi-objective swarm intelligence approach are presented to design optimal ideotypes for sustainable fruit production systems. Both algorithms are successfully applied for generating efficient Pareto optimal solutions in the peach fruit quality improvement problem. The performance of NSGA-II is compared with that of MOPSO-CD and it is found that NSGA-II provides a wide spread of Pareto optimal with better convergence than MOPSO-CD. In addition to this, a 
fuzzy based mechanism is used to find out a compromised solution on the Pareto optimal front.

\section{REFERENCES}

J.E. Fieldsend and Singh, S.: A multi-objective algorithm based upon particle swarm optimization, an efficient data structure and turbulence. In Proc. U.K. Workshop on Computational Intelligence, Birmingham, U.K., (2002) 37-44.

C.A.C. Coello, G.T. Pulido \& M.S. Lechuga. Handling multiple objectives with particle swarm optimization IEEE Transactions on Evolutionary Computation, 8(3), (2OO4) 256-279.

K. Deb. Multi-Objective Optimization using Evolutionary Algorithms (1st edition ed.). New York: Wiley, (2001).

K. Deb, S. Agrawal, A. Pratab \& T. Meyarivan. A fast elitist nondominated sorting genetic algorithm for multiobjective optimization: NSGA II. IEEE Transactions on Evolutionary Computation, 6, (2002) 182-197.

R. Eberhart \& J. Kennedy. A new optimizer using particle swarm theory. Paper presented at the 6th international symposium on micro machine and human science, IEEE service center, Piscataway, NJ, Nagoya, Japan (1995).

M. Génard, N. Bertin, C. Borel, P. Bussieres, H. Gautier, R. Habib et al. Towards a virtual fruit focusing on quality: modelling features and potential uses. Journal of Experimental Botany, 58(5), (2007) 917-928.

M. Génard, N. Bertin, H. Gautier, F. Lescourret \& B. Quilot. Virtual profiling: a new way to analyse phenotypes. Plant Journal, 62(2), (2010) 344-355.

G.L. Hammer, J.W. Hansen, J.G. Phillips, J.W. Mjelde, H. Hill, A. Love et al. Advances in application of climate prediction in agriculture. Agricultural Systems, 70(2-3), (2001) 515-553.

J. Horn, N. Nafploitis \& D.E. Goldberg. A niched Pareto genetic algorithm for multiobjective optimization. Presented at the first IEEE Conference on Evolutionary Computation (1994).

J. Knowles \& D. Corne. The pareto archived evolution strategy: A new baseline algorithm for multiobjective optimization. Presented at the congress on Evolutionary Computation (1999).

F. Lescourret \& M. Génard. A virtual peach fruit model simulating changes in fruit quality during the final stage of fruit growth. Tree Physiology, 25(10), (2005) 13031315.

X. Li. A non-dominated sorting particle swarm optimizer for multiobjective optimization. Proc. of the international conference on Genetic and evolutionary computation: Part I, (2003).

D.G. Mayer. Evolutionary algorithms and agricultural systems. Dordrecht Kluwer Academic Publishers (2002).

B. Panigrahi, V.R. Pandi, R. Sharma, S.Das. Multi-objective bacteria foraging algorithm for electrical load dispatch problem. Energy conversion and management 52 (2) (2011) 1334-1342.

B. Quilot-Turion, M.M. Ould-Sidi, A. Kadrani, N. Hilgert, M. Génard \& F. Lescourret. Optimization of parameters of the 'Virtual Fruit' model to design peach genotype for sustainable production systems. European journal of agronomiy, 42, (2012) 34-48.

C. Raquel \& P. Naval. An effective use of crowding distance in multiobjective particle swarm optimization. Presented at the Conference on genetic and evolutionary computation, Washington (DC, USA) (2005). 
M. Reyes-Sierra \& C.A.C. Coello. Multi-Objective Particle Swarm Optimizers: A Survey of the State-of-the-Art. International Journal of Computational Intelligence Research, 2(3) (2006).

J. R. Schott. Fault tolerant design using single and multicriteria genetic algorithm optimization. Massachusetts Institute of Technology (1995).

N. Srinivas \& K. Deb. Multi-objective optimization using non-dominated sorting in genetic algorithms, Tech. Rep., Indian Institute of Technology, Delhi, India, (1993).

E. Zitzler, K. Deb \& L. Thiele. Comparison of multiobjective evolutionary algorithms: Empirical results. Evolutionary Computation, 8(2), (2000) 173-195.

E. Zitzler \& L. Thiele. An evolutionary algorithm for multiobjective optimization: The strength Pareto approach (Technical Report No. 43). CH-8092 Zurich: Computer Engineering and Networks Laboratory (TIK), Swiss Federal Institute of Technology (ETH), Gloriastrass (1998).

E. Zitzler, M. Laumanns \& L. Thiele. SPAE2: Improving the strength Pareto Evolutionary Algorithm (Technical Report No. 103). CH-8092 Zurich: Computer Engineering and Networks Laboratory (TIK), Swiss Federal Institute of Technology (ETH), Gloriastrass (2001). 